\title{
NSAIDs and Kelleni's protocol as potential early COVID-19 treatment game changer: could it be the final countdown?
}

\author{
Mina T. Kelleni ${ }^{1}$ (D)
}

Received: 26 September 2021 / Accepted: 14 November 2021 / Published online: 25 November 2021

(c) The Author(s), under exclusive licence to Springer Nature Switzerland AG 2021

\begin{abstract}
We have previously published several papers illustrating numerous immunomodulatory and anti-inflammatory potential benefits when we repurposed safe, generic non-steroidal anti-inflammatory drugs (NSAIDs)/nitazoxanide/azithromycin (Kelleni's protocol), to early manage our COVID-19 pediatric, adult, and pregnant patients. In this manuscript, we discuss some recently published meta-analysis and clinical studies supporting our practice and discuss a molecular study that might be interpreted as an academic proof that our protocol might also prevent SARS-CoV-2 replication. Moreover, after aspirin has been suggested to be independently associated with reduced risk of mechanical ventilation, ICU admission and inhospital mortality of COVID-19, we claim that the molecular interpretation of the results that led to this suggestion was not scientifically accurate, and we provide our academic interpretation confirming that low-dose aspirin is least likely to improve COVID-19 mortality through anticoagulation as was suggested. Furthermore, we describe other potential benefits related to aspirin-triggered lipoxins and resolvins while illustrating how NSAIDs interfere with COX-1, COX-2, SARS-CoV-2/ SARS-CoV-2 ORF protein-dependent activation of caspases and their subsequent mitochondrial dysfunction, endoplasmic reticulum stress, apoptosis and necroptosis which were associated with COVID-19 complications. Similarly, NSAIDs are known caspase inhibitors and thus they might independently inhibit other caspase-related COVID-19-associated downstream pathological signaling mechanisms. Finally, we postulated that CARD-14, a caspase recruitment domain-containing protein, polymorphisms might play a role in the development of severe and critical COVID-19 and confirmed our old call to early adopt NSAIDs, as an integral part of Kelleni's protocol, as of choice in its management aiming to end this pandemic.
\end{abstract}

Keywords COVID-19 $\cdot$ SARS-CoV-2 $\cdot$ NSAIDs $\cdot$ Nitazoxanide $\cdot$ Azithromycin $\cdot$ Kelleni's protocol $\cdot$ Aspirin $\cdot$ Caspases $\cdot$ Apoptosis $\cdot$ Endoplasmic reticulum stress

\section{Introduction}

With over five million global deaths, COVID-19 has suddenly and dramatically struck our life, not only as regards to its huge medical consequences, but in many other aspects including social, economic, and political unprecedented conflicts. Unfortunately, it is continuing to daily waste thousands of precious lives all over the world and thus, the quest for a safe pharmacotherapy that could end this pandemic is considered a top global priority. Our approach to manage COVID-19 started in March 2020, when we sent a correspondence to NEJM (20-06753) to refute the claims that

Mina T. Kelleni

mina.kelleni@mu.edu.eg

1 Pharmacology Department, College of Medicine, Minia University, Minya, Egypt led the world to avoid using non-steroidal anti-inflammatory drugs (NSAIDs) to manage COVID-19 patients, calling these claims a scam that lacked any scientific evidence. It was rejected, without a single peer review opportunity, by NEJM and numerous other journals, yet evolved for 5 months until InflammoPharmacology decided to send it for peer review and was eventually published with a more appropriate title (Kelleni 2020a). Fortunately, another paper was fast tracked and was published in April 2020 as we advocated repurposing nitazoxanide/azithromycin to early manage COVID-19 and interestingly, we preferred nitazoxanide over ivermectin as regards to safety and efficacy (Kelleni 2020b).

Since that time, our full protocol repurposing NSAIDs/ nitazoxanide/azithromycin, which we, for sake of simplification, will call it as Kelleni's protocol, used to early manage COVID-19 was clinically practiced and evolving while 
using a personalized medicine approach to manage pediatric, adult and pregnant COVID-19 patients with remarkable safety and efficacy. Notably, numerous researchers supported early management for COVID-19 though differences occurred about which drugs to repurpose and why. We have published several articles that advocated potent anti-inflammatory and immunomodulatory effects for our repurposed drugs with a potential to prevent or restore the immune dysregulation that is well described in COVID-19 (Kelleni 2021a, b, c, d). Interestingly, numerous clinical and academic studies agreed with and supported the safety and efficacy of COVID-19 Kelleni's protocol, though separately for its drugs, as was cited in our previous publications.

In this manuscript, we will discuss and analyze some molecular, clinical, and meta-analysis studies that further support our protocol while adding more insights towards novel molecular and immunomodulatory targets that could add to the mechanisms by which our safe and economic Kelleni's protocol exert its potential COVID-19 game-changer effects.

\section{Kelleni's protocol is safe to manage COVID-19}

Moore et al. have recently published a comprehensive systematic review and meta-analysis that confirmed the safety to use NSAIDs in COVID-19 (Moore et al. 2021). They have confirmed an earlier published one (Kow and Hasan 2021) and a third preprinted article with similar findings (Zhou et al. 2021). Interestingly, a large study by Drake et al. (2021) has urged policy-makers to review their advice regarding prescribing NSAIDs in COVID-19 and some colleagues have recently suggested that earliest COVID-19 home therapy with NSAIDs may greatly prevent hospitalizations (Consolaro et al. 2021; Pandolfi and Chirumbolo 2021). As early mentioned, all these observations and recommendations side with our old one that started since May 2020 when we started to recommend adopting NSAIDs as lifesaving and of choice in COVID-19 management, unless contraindicated due to other reasons or diseases.

Similarly, both the safety and efficacy of nitazoxanide and azithromycin when repurposed to manage COVID-19 are also currently well established and supported by numerous reputable authors who have been cited in our recently published articles (Kelleni 2021b, d) as well as in others (Blum et al., 2021; Cadegiani et al. 2021; Lokhande and Devarajan 2021; Meneses Calderon et al. 2020; Patel et al. 2021).

\section{Kelleni's protocol might inhibit SARS-CoV-2 replication}

Interestingly, a recent research has revealed the vital role played by NF-KB signaling in SARS-CoV-2 replication while insightfully reasoning for the concomitant diminished type I interferon response (Nilsson-Payant Benjamin et al. 2021). In their research, the authors experimentally tested several compounds that disrupted NF- $\mathrm{KB}$ signaling, yet they repeatedly stated that there are no specific FDA-approved drugs that inhibit NF-KB (Nilsson-Payant Benjamin et al. 2021). We would like to confirm that some non-specific FDA-approved drugs augment our natural interferon response while inhibiting and disrupting NF- $\mathrm{KB}$ signaling, including our repurposed Kelleni's immunomodulatory COVID-19 protocol that was shown to restore homeostasis of the SARS-CoV-2 inhibited and/or dysregulated interferon response (Kelleni, 2020b, 2021a, b, c) as well as to inhibit the concomitant SARS-CoV-2-induced robust activation of the IL-6 amplifier including NF- $\mathrm{KB}$ signaling as an essential component (Smart et al. 2020; Kelleni 2021b) which was also showed by Nilsson-Payant and colleagues to play a fundamental role in SARS-CoV-2 replication (NilssonPayant Benjamin et al. 2021). Moreover, we suggest that azithromycin might also disrupt the NF- $\mathrm{KB}$ and signal transducer and activator of transcription- 1 synergistic induction of inflammatory genes expression that could also play a role in the dysregulated COVID-19 immune response (Hiroi and Ohmori 2005; Xing and Liu 2021).

Notably, safe nutraceuticals like resveratrol and quercetin are also known to counteract the inflammatory process partly through modulation of microRNAs which are involved in regulation of NF-kB signaling (Cione et al. 2020) and were suggested to be of potential value in COVID-19 management (Kelleni 2021e; Manjunath and Thimmulappa 2021).

\section{Low-dose aspirin is not the best NSAID to manage COVID-19}

Interestingly, a retrospective cohort study demonstrated that low-dose aspirin, a prototype of NSAIDs, use was independently associated with reduced risk of mechanical ventilation, ICU admission and in-hospital mortality while there were no differences in major bleeding or overt thrombosis between aspirin and non-aspirin users (Chow et al. 2021). However, we suggest that this study has some major flaws in its interpretation and should be properly interpreted from a pathophysiologic and pharmacologic point of view for the best interests of the prospective medical research and more importantly for the welfare of our precious COVID-19 patients. Thus, we will discuss some novel insights about the role that low-dose aspirin, and more clinically importantly other NSAIDs, might play in management of COVID-19 which might include aspirin triggered lipoxins and resolvins, inhibition of cyclooxygenases, SARS-CoV-2 ORF proteins induced caspase activation, necroptosis and endoplasmic reticulum stress aiming at further exploration of COVID-19 
pathophysiology that might guide us in our vigorous quest for a highly anticipated cure.

Chow et al. have cited numerous references that correlated with SARS-CoV-2-induced hypercoagulable state and subsequent development of platelet rich thrombi with severe COVID-19 and mortality and they have cited a study performed by Paranjpe et al. (2020) which has suggested that systemic treatment-dose anticoagulation may be associated with improved outcomes among hospitalized COVID19 patients to suggest that their reported aspirin beneficial outcomes might be due to its well-known antithrombotic properties. However, Paranjpe et al. have clearly enumerated numerous limitations of their study, and the effect of the prophylactic low-dose aspirin tested by Chow et al. might differ from that of the systemic treatment dose anticoagulants studied by Paranjpe et al. To be also noted is that a large observational study has demonstrated no significant association between ongoing use of direct oral anticoagulants and severe COVID-19 and wisely suggested that therapies should be better directed against thrombogenic inflammation, the cause, rather than against hypercoagulability, the symptom (Flam et al. 2021). Similarly, a systematic review of literature supported a similar conclusion while criticizing a low scientific quality of the described studies (Tieleman et al. 2021). Importantly, Chow et al. have not found a difference in incidence of overt thrombosis between aspirin and non-aspirin users and thus, we suggest that sub-overt mechanisms, discussed in the following paragraphs, should be attributed to reason for the potential aspirin beneficial effects in COVID-19 as expressed by Chow et al.

Moreover, Chow et al. have stated that aspirin, as a cyclooxygenase-1 (COX-1) inhibitor, modifies both inflammatory and coagulation responses and they cited a review written by Warner et al. (2011). However, in that cited reference, no mention to a link between COX-1 inhibition and inflammation was found and it was clearly stated, at that reference as elsewhere, that COX-1 is the constitutive form of the enzyme which is also exclusively or dominantly expressed in the anucleated platelets and that COX-2 is the inducible one associated with inflammation. Similarly, Chow et al. have cited a resourceful review and meta-analysis written by Panka et al. (2017) to reason for the aspirin's anti-inflammatory mechanisms including lipoxin formation. However, in that reference, these mechanisms were evident in murine or in in vitro preclinical models and in some of which aspirin was applied by local administration and in all these models, aspirin was used, as also stated, in high doses in contrast to the low doses used in clinical studies including that of Chow et al. and thus the evidence cited from Panka et al. does not reason for Chow et al. aspirin's anti-inflammatory properties. Additionally, Panka et al. discussed some contradictory results found in sheep and murine models and Chow et al. have also wisely confirmed that aspirin showed mixed results when tested for acute respiratory distress syndrome and cited few studies though only thoroughly discussed the positive ones.

Furthermore, Chow et al. have also cited a study performed by Ikonomidis et al. (1999) in which $300 \mathrm{mg}$ daily aspirin was administered for 6 weeks and decreased IL- 6 and CRP to reflect on their $81 \mathrm{mg}$ aspirin dose and this reflection is also not scientifically justified as low-dose aspirin cannot inhibit the inflammatory COX-2, as stated by Chow et al., and inhibits COX-1 almost selectively. Moreover, Ikonomidis et al. have also mentioned that aspirin exhibits anti-inflammatory action in a dose-dependent manner and its greatest effects occur at doses as high as $2 \mathrm{~g}$.

\section{Potential benefits of low-dose aspirin in COVID-19}

In our opinion, the results presented by Chow et al. should be interpreted and built upon by researching potential aspirin's non-COX-dependent anti-inflammatory effects through modulation of the immune and inflammatory function of platelets (Sonmez and Sonmez 2017; Kasirer-Friede et al. 2019) as well as its peculiar ability to trigger induction of the beneficial anti-inflammatory and immunomodulatory lipoxins and resolvins which are synthesized through acetylated COX-2 (Serhan et al. 2008).

Notably, while COX-2 acetylation, and the subsequent formation of lipoxins and resolvins, is not achievable by lowdose aspirin, induction of COX-1 upregulation in COVID19 might be considered for further research as it has been previously described, with potential benefits of its inhibition under certain conditions, in some neuroinflammatory and neurodegenerative diseases. Additionally, COX-1 and/or COX-2 potential role in SARS-CoV-2 replication should be assessed and NSAIDs were also suggested, in a preprint, to directly affect SARS-CoV-2 replication (Chen et al. 2020).

\section{NSAIDs potential modulation of SARS-CoV-2-induced activation of caspases, apoptosis, and necroptosis}

Caspases are a family of enzymes associated with apoptosis, pyroptosis and their dysregulated activation was suggested to share in the pathogenesis of tumors, autoimmune, autoinflammatory, inflammatory cytokine secretion including IL-1 $\beta$ from viable monocytes as well as infectious disorders (Van Opdenbosch and Lamkanfi 2019). SARS-CoV-2 infection was reported to activate caspase-8-triggering proinflammatory cytokines, including IL- $1 \beta$, TNF- $\alpha$, IL-7, IL-8, apoptosis, necroptosis and activation of the NFKB pathway in lung epithelial cells which were suggested to 
share in COVID-19-induced downstream immune pathogenesis causing lung damage ( $\mathrm{Li}$ et al. 2020). Moreover, open reading frames (ORF) 3a protein of SARS-CoV-2 was shown to significantly induce cellular apoptosis which was shown experimentally to be significantly inhibited by either a caspase 8 or caspase 9 inhibitor (Ren et al. 2020) to be noted that several ORF SARS-CoV proteins were previously shown to induce apoptosis and ORF-6 protein overexpression was shown to induce caspase-3 mediated c-Jun N-terminal kinase (JNK)-dependent apoptosis that was blocked by a specific caspase 3 inhibitor or JNK inhibitor (Ye et al. 2008). Similarly, caspase-mediated apoptosis was suggested to play a significant role in COVID-19 complications (Fakhri et al. 2021), and blocking caspase activity was shown to in vitro rescue $\mathrm{T}$ cells by inhibiting their apoptosis and thus, inhibiting caspases could play a role in severe COVID-19 which is characterized by dysfunctional immune response (Thompson et al. 2021). Interestingly, several caspases were reported to modulate $\mathrm{B}$ and $\mathrm{T}$ cell proliferation and altered transcriptome levels of caspase genes were reported in natural killer cells and neutrophils. Moreover, uncontrolled caspase response in COVID-19 was suggested to share the immune pathological processes as well as in the inflammatory microvascular thrombi found in multiple organs leading to severe outcomes. Interestingly, caspase- 1 in CD4 + T cells was shown to be upregulated in hospitalized COVID19 patients and caspase-3 levels were reported to be significantly upregulated, compared to controls, in circulating red blood cells from COVID-19 patients as well as in tissue macrophages in postmortem analysis and were also demonstrated to be suppressed ex vivo by a pan caspase inhibitor (Plassmeyer et al. 2020). Thus, unsurprisingly, suppression of apoptosis was suggested to prevent viral pathogenesis in some diseases including SARS and targeting virus-induced apoptosis was implied as a promising strategy in COVID-19 management (Donia \& Bokhari, 2021, though sometimes drugs with unbalanced risk:benefit ratio were suggested (Fakhri et al. 2021). Similarly, inhibition of the necroptosis signaling pathway, a subsequent outcome of caspase- 8 activation, was suggested to possess a potential to protect against COVID-19 complications (Cao and Mu 2021).

Notably, we postulate that CARD 14, a caspase recruitment domain-containing protein of the membrane-associated guanylate kinases family https://www.ncbi.nlm.nih.gov/ gene/79092, mutations might play a crucial role in COVID19 pathogenesis and complications especially in severe and critical patients and we further suggest that the quest to design and develop novel caspase inhibitors and/or modulators might evolve to be corner stone in management of several immune-inflammatory diseases,

Importantly, we would like to pharmacologically confirm that NSAIDs are well known, at physiologic in vivo concentrations, caspase inhibitors (Smith et al. 2017) and we suggest they are the safest drugs among all the suggested and investigated caspase inhibitors (Aranda et al. 2017).

\section{NSAIDs potential modulation of SARS-CoV-2 induced endoplasmic reticulum stress}

Furthermore, endoplasmic reticulum stress (ERS) was suggested to play an important role in the development of COVID-19 (Banerjee et al. 2020) and ERS markers were shown to be significantly increased in SARS-CoV-2 infection and COVID-19 pneumonia (KÖSeler et al. 2020). Interestingly, several NSAIDs including diclofenac, indomethacin, ibuprofen, aspirin, and ketoprofen were shown to suppress the ERS-induced human neuroblastoma SH-SY5Y cell death (Yamazaki et al. 2006). Similarly, oxicam-derived NSAIDs have been demonstrated to possess neuroprotective effects potentially through suppressed activation of caspase-3 and cell death as well as amelioration of ERS and/or mitochondrial dysfunction signaling pathways (Omura et al. 2018) and thus, NSAIDs potential direct and indirect positive immunomodulatory effects in COVID-19 are further amplified through their potential anti-ERS effects.

\section{Potential therapeutic role of Kelleni's protocol in COVID-19}

Taken together, we wish to highlight that overwhelming pieces of evidence should urge policy-makers to finally consider old recommendation to use NSAIDs as of choice in the management of COVID-19 and though we previously and relentlessly called for almost half past a year for large clinical trials to test Kelleni's protocol, we currently suggest that it might be more appropriate to adopt it soonest as proven safe and effective by numerous researchers who tested its used repurposed drugs, separately. Our call is aimed to save precious lives that have been and are being deprived safe, economic generic repurposed drugs especially as COVID-19 is re-surging in the European countries to the extent that the WHO has recently described them again as the epicenter for the pandemic [https://www.euro.who.int/en/health-topics/ health-emergencies/coronavirus-covid-19/statements/state ment-update-on-covid-19-europe-and-central-asia-againat-the-epicentre-of-the-pandemic/] and we recommend adopting therapeutic doses of NSAIDs in the management of COVID-19 as explained in our published manuscripts if some authorities decided to simultaneously perform sufficiently powered randomized clinical trials against any standard protocol.

We have previously postulated that NSAIDs, as integral part of Kelleni's protocol, are potentially lifesaving intervention tools to prevent or reverse the evolution of the 
well-described aggressive hyperinflammatory responses in COVID-19 (Kelleni, 2020a, 2021a) due to their immunomodulatory and anti-inflammatory properties (Kelleni 2021b) and in this regard, NSAIDs might possess a huge advantage as, unlike corticosteroids (Kelleni 2021f), they are not immunosuppressive. Moreover, we demonstrated our real-life personalized approach that adopts NSAIDs as an integral part of COVID-19 management (Kelleni 2021d). However, we recommend against the concomitant use of prophylactic low-dose aspirin and NSAIDs, or at least some of them (Gurbel et al. 2019), and we obviously opt for NSAIDs over low-dose aspirin.

Finally, we wish it might be the time for final countdown to return our world, our lives and our gatherings back to safety and we will never ask what if, it is not practical, but instead we will keep asking when will all policy-makers start to consider that a safe, economic, and highly effective potential COVID-19 curative protocol can end this pandemic?

Acknowledgements I wish to express my utmost gratitude and respect to InflammoPharmacology, its honorable editor in chief Prof. Dr. Kim D. Rainsford and editorial board for giving my manuscripts a very precious opportunity to be properly peer reviewed. I am also indebted to honorable anonymous peer reviewers whose highly constructive insightful remarks have significantly improved the quality of the presented manuscript.

Author contributions Sole author.

Funding None.

\section{Declarations}

Conflict of interest The author has no conflicts of interest to declare.

Statement of ethics Not applicable.

\section{References}

Aranda JV, Salomone F, Valencia GB, Beharry KD (2017) Non-steroidal anti-inflammatory drugs in newborns and infants. Pediatr Clin North Am 64:1327-1340

Banerjee A, Czinn SJ, Reiter RJ, Blanchard TG (2020) Crosstalk between endoplasmic reticulum stress and anti-viral activities: a novel therapeutic target for COVID-19. Life Sci 255:117842

Blum VF, Cimerman S, Hunter JR, Tierno P, Lacerda A, Soeiro A et al (2021) Nitazoxanide superiority to placebo to treat moderate COVID-19: a pilot prove of concept randomized double-blind clinical trial. EClinicalMedicine 37:100981

Cadegiani FA, Goren A, Wambier CG, McCoy J (2021) Early COVID19 therapy with azithromycin plus nitazoxanide, ivermectin or hydroxychloroquine in outpatient settings significantly improved COVID-19 outcomes compared to known outcomes in untreated patients. New Microbes New Infect 43:100915

Cao L, Mu W (2021) Necrostatin-1 and necroptosis inhibition: pathophysiology and therapeutic implications. Pharmacol Res 163:105297-105297
Chen JS, Alfajaro MM, Wei J, Chow RD, Filler RB, Eisenbarth $\mathrm{SC}$ et al (2020) Cyclooxgenase-2 is induced by SARS-CoV-2 infection but does not affect viral entry or replication. bioRxiv. https://doi.org/10.1101/2020.09.24.312769

Chow JH, Khanna AK, Kethireddy S, Yamane D, Levine A, Jackson AM et al (2021) Aspirin use is associated with decreased mechanical ventilation, intensive care unit admission, and inhospital mortality in hospitalized patients with coronavirus disease 2019. Anesth Analg 132(4):930-941

Cione E, La Torre C, Cannataro R, Caroleo MC, Plastina P, Gallelli L et al (2020) Quercetin, epigallocatechin gallate, curcumin, and resveratrol: from dietary sources to human MicroRNA Modulation. Molecules 25:63-63

Consolaro E, Suter F, Rubis N, Pedroni S, Moroni C, Pastò E et al (2021) A home-treatment algorithm based on anti-inflammatory drugs to prevent hospitalization of patients with early covid- 19 . medRxiv. https://doi.org/10.1101/2021.09.29.21264298

Donia A, Bokhari H (2021) Apoptosis induced by SARS-CoV-2: can we target it? Apoptosis 26:7-8

Drake TM, Fairfield CJ, Pius R, Knight SR, Norman L, Girvan M et al (2021) Non-steroidal anti-inflammatory drug use and outcomes of COVID-19 in the ISARIC Clinical Characterisation Protocol UK cohort: a matched, prospective cohort study. Lancet Rheumatol 3:e498-e506

Fakhri S, Nouri Z, Moradi SZ, Akkol EK, Piri S, Sobarzo-Sanchez E et al (2021) Targeting multiple signal transduction pathways of sars-CoV-2: approaches to COVID-19 therapeutic candidates. Molecules 26(10):2917

Flam B, Wintzell V, Ludvigsson JF, Mårtensson J, Pasternak B (2021) Direct oral anticoagulant use and risk of severe COVID19. J Intern Med 289:411-419

Gurbel P, Tantry U, Weisman S (2019) A narrative review of the cardiovascular risks associated with concomitant aspirin and NSAID use. J Thromb Thrombolysis 47:16-30

Hiroi M, Ohmori Y (2005) Transcriptional synergism between NF- $\kappa$ B and STAT1. J Oral Biosci 47:230-242

Ikonomidis I, Andreotti F, Economou E, Stefanadis C, Toutouzas P, Nihoyannopoulos P (1999) Increased proinflammatory cytokines in patients with chronic stable angina and their reduction by aspirin. Circulation 100:793-798

Kasirer-Friede A, Tjahjono W, Eto K, Shattil SJ (2019) SHARPIN at the nexus of integrin, immune, and inflammatory signaling in human platelets. Proc Natl Acad Sci U S A 116:4983-4988

Kelleni MT (2020a) ACEIs, ARBs, ibuprofen originally linked to COVID-19: the other side of the mirror. Inflammopharmacology $28: 1477-1480$

Kelleni MT (2020b) Nitazoxanide/azithromycin combination for COVID-19: A suggested new protocol for early management. Pharmacol Res 2020(157):104874. https://doi.org/10.1016/j. phrs.2020.104874 (Epub 2020 Apr 30. PMID: 32360581; PMCID: PMC7192107)

Kelleni MT (2021a) Early use of non-steroidal anti-inflammatory drugs in COVID-19 might reverse pathogenesis, prevent complications and improve clinical outcomes. Biomed Pharmacother 133:110982

Kelleni MT (2021b) NSAIDs/Nitazoxanide/azithromycin repurposed for COVID-19: potential mitigation of the cytokine storm interleukin-6 amplifier via immunomodulatory effects. Expert Rev Anti Infect Ther. https://doi.org/10.1080/147872102021193 9683

Kelleni MT (2021c) COVID-19, Ebola virus disease, and Nipah virus infection reclassification as novel acute immune dysrhythmia syndrome (n-AIDS): potential crucial role for immunomodulators. Immunol Res 69(5):457-460

Kelleni MT (2021d) NSAIDs/Nitazoxanide/azithromycin immunomodulatory protocol used in adult, geriatric, pediatric, pregnant, and 
immunocompromised COVID-19 patients: a real-world experience. Can J Med 3(3):121-143

Kelleni MT (2021e) Resveratrol-zinc nanoparticles or pterostilbenezinc: Potential COVID-19 mono and adjuvant therapy. Biomed Pharmacother 139:111626

Kelleni MT (2021f) Tocilizumab, remdesivir, favipiravir, and dexamethasone repurposed for COVID-19: a comprehensive clinical and pharmacovigilant reassessment. SN Compr Clin Med 3:919-923

KÖSeler A, Sabirli R, GÖren T, TÜrkçÜEr İ, Kurt Ö (2020) Endoplasmic reticulum stress markers in SARS-COV-2 infection and pneumonia: case-control study. In Vivo 34:1645

Kow CS, Hasan SS (2021) The risk of mortality in patients with COVID-19 with pre-diagnosis use of NSAIDs: a meta-analysis. Inflammopharmacology 29:641-644

Li S, Zhang Y, Guan Z, Li H, Ye M, Chen X et al (2020) SARS-CoV-2 triggers inflammatory responses and cell death through caspase- 8 activation. Signal Transduct Target Ther 5:235

Lokhande AS, Devarajan PV (2021) A review on possible mechanistic insights of Nitazoxanide for repurposing in COVID-19. Eur J Pharmacol 891:173748

Manjunath SH, Thimmulappa RK (2021) Antiviral, immunomodulatory, and anticoagulant effects of quercetin and its derivatives: Potential role in prevention and management of COVID-19. J Pharm Anal. https://doi.org/10.1016/j.jpha.2021.09.009

Meneses Calderon J, Figueroa Flores MDR, Paniagua Coria L, Briones Garduno JC, Meneses Figueroa J, Vargas Contretas MJ et al (2020) Nitazoxanide against COVID-19 in three explorative scenarios. J Infect Dev Ctries 14:982-986

Moore N, Bosco-Levy P, Thurin N, Blin P, Droz-Perroteau C (2021) NSAIDs and COVID-19: a systematic review and meta-analysis. Drug Saf 44:929-938

Nilsson-Payant Benjamin E, Uhl S, Grimont A, Doane Ashley S, Cohen P, Patel Roosheel S et al (2021) The NF- $\kappa B$ transcriptional footprint is essential for SARS-CoV-2 replication. J Virol 95(23): 0125721

Omura T, Sasaoka M, Hashimoto G, Imai S, Yamamoto J, Sato Y et al (2018) Oxicam-derived non-steroidal anti-inflammatory drugs suppress 1-methyl-4-phenyl pyridinium-induced cell death via repression of endoplasmic reticulum stress response and mitochondrial dysfunction in SH-SY5Y cells. Biochem Biophys Res Commun 503:2963-2969

Pandolfi S, Chirumbolo S (2021) Home therapy of COVID-19 at the earliest may greatly prevent hospitalization. Basic Clin Pharmacol Toxicol. https://doi.org/10.1111/bcpt.13650

Panka BA, de Grooth H-J, Spoelstra-de Man AME, Looney MR, Tuinman P-R (2017) Prevention or treatment of Ards with aspirin: a review of preclinical models and meta-analysis of clinical studies. Shock 47:13-21

Paranjpe I, Fuster V, Lala A, Russak AJ, Glicksberg BS, Levin MA et al (2020) Association of treatment dose anticoagulation with in-hospital survival among hospitalized patients with COVID-19. J Am Coll Cardiol 76:122-124

Patel J, Berezowski I, Abdelmonem A, Taylor D, Pourmand A (2021) Azithromycin for mild-to-moderate COVID-19. Lancet Respir Med 9:e99
Plassmeyer M, Alpan O, Corley MJ, Lillard K, Coatney P, Vaziri T et al (2020) Caspases in COVID-19 disease and sequela and the therapeutic potential of caspase inhibitors. medRxiv. https://doi. org/10.1101/2020.11.02.20223636

Ren Y, Shu T, Wu D, Mu J, Wang C, Huang M et al (2020) The ORF3a protein of SARS-CoV-2 induces apoptosis in cells. Cell Mol Immunol 17:881-883

Serhan CN, Chiang N, Van Dyke TE (2008) Resolving inflammation: dual anti-inflammatory and pro-resolution lipid mediators. Nat Rev Immunol 8:349-361

Smart L, Fawkes N, Goggin P, Pennick G, Rainsford KD, Charlesworth B et al (2020) A narrative review of the potential pharmacological influence and safety of ibuprofen on coronavirus disease 19 (COVID-19), ACE2, and the immune system: a dichotomy of expectation and reality. Inflammopharmacology 28:1141-1152

Smith CE, Soti S, Jones TA, Nakagawa A, Xue D, Yin H (2017) Nonsteroidal anti-inflammatory drugs are caspase inhibitors. Cell Chem Biol 24:281-292

Sonmez O, Sonmez M (2017) Role of platelets in immune system and inflammation. Porto Biomed J 2:311-314

Thompson EA, Cascino K, Ordonez AA, Zhou W, Vaghasia A, Hamacher-Brady A et al (2021) Metabolic programs define dysfunctional immune responses in severe COVID-19 patients. Cell Rep 34:108863

Tieleman RG, Klok FA, Belfroid E, Hoogervorst-Schilp J, Schalkers I, Jansen CW et al (2021) Effect of anticoagulant therapy in COVID19 patients. Neth Heart J 29:35-44

Van Opdenbosch N, Lamkanfi M (2019) Caspases in cell death, inflammation, and disease. Immunity 50:1352-1364

Warner TD, Nylander S, Whatling C (2011) Anti-platelet therapy: cyclo-oxygenase inhibition and the use of aspirin with particular regard to dual anti-platelet therapy. Br J Clin Pharmacol 72:619-633

Xing YW, Liu KZ (2021) Azithromycin inhibited oxidative stress and apoptosis of high glucose-induced podocytes by inhibiting STAT1 pathway. Drug Dev Res 82:990-998

Yamazaki T, Muramoto M, Oe T, Morikawa N, Okitsu O, Nagashima T et al (2006) Diclofenac, a non-steroidal anti-inflammatory drug, suppresses apoptosis induced by endoplasmic reticulum stresses by inhibiting caspase signaling. Neuropharmacology 50:558-567

Ye Z, Wong CK, Li P, Xie Y (2008) A SARS-CoV protein, ORF-6, induces caspase-3 mediated, ER stress and JNK-dependent apoptosis. Biochim Biophys Acta 1780:1383-1387

Zhou Q, Zhao S, Gan L, Wang Z, Peng S, Li Q et al (2021) Associations between the use of non-steroidal anti-inflammatory drugs and adverse outcomes among patients with COVID-19: a systematic review and meta-analysis. SSRN. https://doi.org/10.2139/ ssrn.3903939

Publisher's Note Springer Nature remains neutral with regard to jurisdictional claims in published maps and institutional affiliations. 